\title{
Scutellarin regulates osteoarthritis in vitro by inhibiting the PI3K/AKT/mTOR signaling pathway
}

\author{
SHAO-HUA JU ${ }^{1}$, LI-RONG TAN ${ }^{2}$, PAN-WANG LIU ${ }^{3}$, YOU-LI TAN ${ }^{1}$, YUAN-TING ZHANG ${ }^{1}$, \\ XIAO-HONG LI ${ }^{1}$, MING-JIAN WANG ${ }^{1}$ and BEN-XIANG HE ${ }^{1}$ \\ ${ }^{1}$ Department of Pharmacy, Sport Hospital Attached of CDSU, Chengdu Sport University, Chengdu, \\ Sichuan 610041; ${ }^{2}$ Department of Internal Medicine, An Jing Countryside Hospital, Chengdu, Sichuan 611730; \\ ${ }^{3}$ Department of Pharmacy, Chengdu University of Traditional Chinese Medicine, Chengdu, Sichuan 611137, P.R. China
}

Received February 14, 2020; Accepted July 14, 2020

DOI: $10.3892 / \mathrm{mmr} .2020 .11722$

\begin{abstract}
Osteoarthritis (OA) is a highly prevalent disease worldwide that causes disability and diminishes the quality of life of affected individuals. The disease is characterized by cartilage destruction, increased inflammatory responses and cholesterol metabolic disorder. Scutellarin is the major active ingredient extracted from Erigeron breviscapus, and it has been demonstrated to possess various pharmacological functions in the treatment of the disease. However, its effects on OA are complex. The present study investigated whether scutellarin can mediate the release of inflammatory cytokines, the expression of collagen- and cholesterol-related proteins, and regulate the phosphoinositide 3-kinase $(\mathrm{PI} 3 \mathrm{~K}) / \mathrm{AKT} / \mathrm{mammalian}$ target of rapamycin (mTOR) signaling pathway in a cell model of OA. Interleukin (IL)-1 $\beta$ was used to stimulate OA in SW1353 cells in vitro. The primary methods used were ELISA and western blotting, which were carried out to examine the effects of scutellarin on the cell model of OA. It was found that scutellarin increased the expression of collagen II and SRY-box 9, whereas it suppressed the expression of matrix metalloproteinase 13. In addition, scutellarin downregulated the expression levels of cholesterol 25-hydroxylase and cytochrome P450 family 7 subfamily B polypeptide 1, but upregulated the expression of apolipoprotein A-1 and adenosine triphosphate-binding cassette transporter A1. The IL-1 $\beta$-induced increase in the expression of IL- 6 was decreased by treatment with scutellarin; however, scutellarin did not alter the expression of $\mathrm{C}$-reactive protein and tumor necrosis factor- $\alpha$. The protein
\end{abstract}

Correspondence to: Dr Ben-Xiang He, Department of Pharmacy, Sport Hospital Attached of CDSU, Chengdu Sport University, $2 \mathrm{Ti}$ Yuan Road, Chengdu, Sichuan 610041, P.R. China

E-mail: 1210075193@qq.com

Key words: osteoarthritis, scutellarin, inflammation, cholesterol, collagen, phosphatidylinositol 3 kinase/protein kinase B/mammalian target of rapamycin signaling pathway expression levels of AKT, phosphorylated (p)-AKT, mTOR and $\mathrm{p}-\mathrm{mTOR}$ in the PI3K/AKT/mTOR signaling pathway were decreased in the IL-1 $\beta$-induced SW1353 cells following scutellarin treatment. Overall, the findings of the present study demonstrated that scutellarin regulated OA in vitro by inhibiting the PI3K/AKT/mTOR signaling pathway.

\section{Introduction}

Osteoarthritis (OA) is the most common degenerative joint disease affecting the articular cartilage worldwide, and causes pain, stiffness and decreased mobility, thus resulting in a reduced quality of life $(1,2)$. It has been demonstrated that $10-18 \%$ of adults $>60$ years of age suffer from OA, and the number of patients with $\mathrm{OA}$ is estimated to increase to $>130$ million worldwide by the year 2050 (3). Factors, such as genetics, age, sex, body mass index, nutrition and joint leptin levels are related to OA (3-5). Initially, OA was considered to be a disease of the cartilage; however, it was subsequently demonstrated to be a complex disease mediated by inflammation (6). Additionally, high cholesterol levels have been found to be closely related to OA (7). Currently, an increasing number of studies are focusing on cholesterol metabolism and inflammation in OA.

The phosphoinositide 3-kinase (PI3K)/AKT/mammalian target of rapamycin (mTOR) signaling pathway is a common signaling pathway that plays a role in the progression of a number of diseases, and therefore may also be involved in the development of OA. As previously reported, the inhibition of the PI3K/AKT/mTOR signaling pathway can attenuate the inflammatory response and promote autophagy in articular chondrocytes in rats (8). The increment of PI3K/AKT signaling activation has been demonstrated to be associated with the degeneration of articular cartilage (9). Thus, the inhibition of the PI3K/AKT/mTOR signaling pathway has been viewed as a treatment strategy for OA (10).

Aside from the inflammatory response, high cholesterol levels are another important risk factor of OA (11). High levels of low-density lipoprotein cholesterol (LDL-C) accelerate the incidence of OA in humans and mice $(12,13)$. The molecular mechanisms are associated with the cholesterol 25-hydroxylase $(\mathrm{CH} 25 \mathrm{H})$ /cytochrome $\mathrm{P} 450$ family 7 subfamily $\mathrm{B}$ 
polypeptide 1 (CYP7B1)/RAR-related orphan receptor $\alpha$ axis. In psoriasis, it has been reported that the activation of the PI3K/AKT/mTOR signaling pathway plays a vital role in increasing cholesterol levels (14). Another study demonstrated that insulin-induced cholesterol uptake, lipid droplet content and apolipoprotein $\mathrm{B}$ secretion in $\mathrm{CaCo}-2$ cells were associated with the PI3K/AKT/mTOR signaling pathway (15). For this reason, the expression levels of cholesterol-related proteins and proteins in the PI3K/AKT/mTOR signaling pathway were measured in the present study.

Chinese herbal medicine can regulate the PI3K/AKT/mTOR signaling pathways by attenuating interleukin (IL)-1 $\beta$-induced apoptosis and extracellular matrix (ECM) catabolism in OA (16). Chinese herbal medicine is frequently used as a therapeutic strategy for OA. Herbs, such as Clematis, Nigella sativa, Radix Angelica sinensis, Salvia miltiorrhiza and the components purified from these herbs have been reported to attenuate the development of $\mathrm{OA}$ or even to function as therapies for OA (17-20). Scutellarin is an active flavonoid isolated from the Chinese traditional herb Erigeron breviscapus, which has been found to possess several functions, such as anti-inflammatory and antioxidant activities (21), and it has also been reported to exert effects on various types of tumors , such as malignant melanoma and bladder cancer $(22,23)$.

The effects of scutellarin in OA have been partially studied; however, its mechanism of action is complex. The present study investigated the function of scutellarin in regulating the release of inflammatory cytokines, the expression of collagen- and cholesterol-related proteins, as well as its effects on the PI3K/AKT/mTOR signaling pathway. IL-1 $\beta$ was used to stimulate human osteosarcoma cells (SW1353) to induce OA in vitro, and the effects of scutellarin with or without a PI3K inhibitor (LY294002) on IL-1ß-induced SW1353 cells were then observed. The results revealed that scutellarin inhibited the expression of IL-6, and regulated the expression levels of collagen-related proteins, collagen II (Col2), SRY-box 9 (SOX9) and matrix metalloproteinase (MMP)13, and cholesterol-related proteins, CH25H, CYP7B1, apolipoprotein A-1 (APOA-1) and adenosine triphosphate-binding cassette transporter A1 (ABCA1). As the expression levels of AKT, phosphorylated (p)-AKT, mTOR and p-mTOR were suppressed by scutellarin treatment, it was thus suggested that scutellarin regulates $\mathrm{OA}$ in vitro by inhibiting the $\mathrm{PI} 3 \mathrm{~K} / \mathrm{AKT} / \mathrm{mTOR}$ signaling pathway.

\section{Materials and methods}

Reagents. Scutellarin was purchased from Shanghai Yuanye Bio-Technology Co., Ltd. The Cell Counting Kit-8 (CCK-8) was obtained from Vazyme Biotech Co., Ltd. LY294002 was purchased from Sigma-Aldrich (Merck KGaA). IL-1 $\beta$ was obtained from Novoprotein Scientific, Inc. RIPA lysate was purchased from Beyotime Institute of Biotechnology. Primary antibodies against Col2 (1:2,000; cat. no. ab34712), SOX9 (1:1,000; cat. no. ab185966), MMP13 (1:1,000; cat. no. ab51072), CH25H (1:500; cat. no. ab133933), CYP7B1 (1:1,000; cat. no. ab138497), ABCA1 (1:200; cat. no. ab18180), APOA-1 (1:500; cat. no. ab75114), PI3K (1:1,000; cat. no. ab32089), AKT (1:500; cat. no. ab8805), p-AKT (1:500; cat. no. ab38449), mTOR (1:1,000; cat. no. ab32028) and p-mTOR (1:500; cat. no. ab84400), and a goat anti-rabbit secondary antibody (1:5,000; cat. no. ab6721) were purchased from Abcam. The primary antibodies and secondary antibodies were used according to the manufacturer's instructions.

Cell culture and treatment. SW1353 cells (The Cell Bank of Type Culture Collection of the Chinese Academy of Sciences) were cultured in Dulbecco's modified Eagle's medium (DMEM; Gibco; Thermo Fisher Scientific, Inc.) supplemented with 10\% FBS (Gibco; Thermo Fisher Scientific, Inc.), $100 \mathrm{units} / \mathrm{ml}$ penicillin and $100 \mu \mathrm{g} / \mathrm{ml}$ streptomycin. The cells were maintained at $37^{\circ} \mathrm{C}$ in $5 \% \mathrm{CO}_{2}$. To assess cell viability, SW1353 cells $\left(7 \times 10^{3}\right.$ cells/well) were cultured in 96 -well plates overnight and then treated with increasing concentrations of scutellarin $(0,5,10,20,40,80$ and $100 \mu \mathrm{mol} / \mathrm{l})$ for $48 \mathrm{~h}$ at $37^{\circ} \mathrm{C}$. For the second part of the experiment, SW1353 cells were pre-treated with $80 \mu \mathrm{mol} / 1$ scutellarin for $2 \mathrm{~h}$, following which IL-1 $\beta(10 \mathrm{ng} / \mathrm{ml})$ was added to the cells for $48 \mathrm{~h}$ at $37^{\circ} \mathrm{C}$. The dose of IL-1 $\beta$ was used as reported by Xue et al (8) and Liu et al (24). Subsequently, $10 \mu 1$ CCK-8 reagent was added to each well for a further $2 \mathrm{~h}$ of incubation, according to the manufacturer's instructions. The absorbance value was assessed using a microplate reader (BioTek Epoch; BioTek Instruments, Inc.) at $450 \mathrm{~nm}$. To inhibit the PI3K/AKT signaling pathway, serum-starved $(0.5 \%$ FBS $)$ cells were pre-treated with scutellarin $(80 \mu \mathrm{mol} / \mathrm{l})$ for $2 \mathrm{~h}$ and LY294002 $(25 \mu \mathrm{g} / \mathrm{ml})$ for $1 \mathrm{~h}$, and then exposed to IL-1 $\beta(10 \mathrm{ng} / \mathrm{ml})$ for $48 \mathrm{~h}$. The test was repeated three times.

ELISA for conditioned medium. The expression levels of C-reactive protein (CRP), tumor necrosis factor (TNF)- $\alpha$ and IL-6 in conditioned medium (collected from culture supernatant) were measured using CRP (cat. no. ZC-31853; ZCi Bio), TNF- $\alpha$ (cat. no. ZC-35733; ZCi Bio) and IL-6 (cat. no. ZC-32446; ZCi Bio) ELISA kits following the manufacturer's instructions.

Western blot analysis. RIPA buffer containing $0.1 \%$ protease inhibitor was used to homogenize the cell samples, and cell lysates were centrifuged at $13,680 \mathrm{x}$ g for $15 \mathrm{~min}$ at $4^{\circ} \mathrm{C}$, and the supernatants were then collected for protein detection. Total protein concentrations were measured using a BCA kit (Thermo Fisher Scientific, Inc.). Equal amounts of protein (20 $\mu \mathrm{g}$ per lane for cell samples) were separated via 10\% SDS-PAGE and then transferred onto PVDF membranes. The membranes were then blocked in 5\% skimmed milk at room temperature for $2 \mathrm{~h}$. After washing in TBST three times, the immunoblots were incubated with the following primary antibodies (3\% BSA dilution): $\mathrm{Col} 2$, SOX9, MMP13, $\mathrm{CH} 25 \mathrm{H}$, CYP7B1, ABCA1, APOA-1, PI3K, AKT, p-AKT, mTOR and p-mTOR (details of antibody dilutions were described in the 'Reagents' section) overnight at $4^{\circ} \mathrm{C}$. The membranes were then incubated with goat anti-rabbit IgG secondary antibody (1:5,000, $1 \%$ BSA dilution) for $2 \mathrm{~h}$ at room temperature. The membranes were washed with TBST again and then detected with ECL (EMD Millipore). The band sizes were quantified using Scion Image 4.0 software (Scion Corporation). Protein expression was normalized relative to $\beta$-actin as a loading control. The final results are expressed as 'fold changes' in comparison with the control group. 

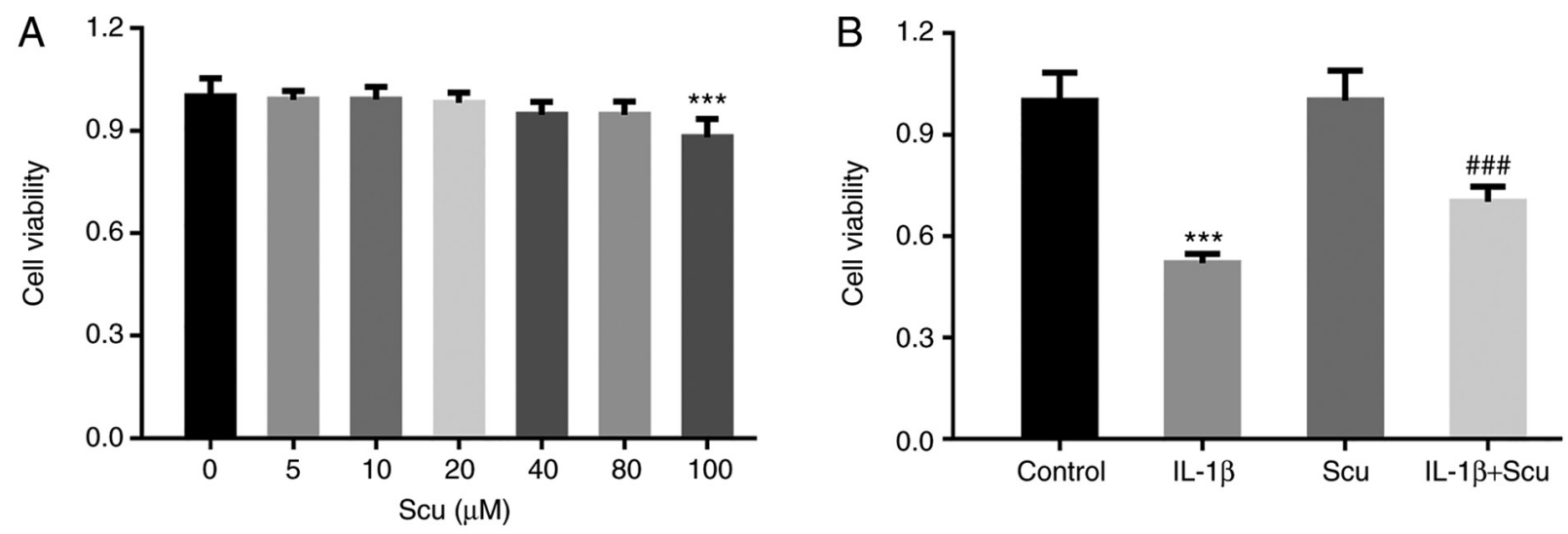

Figure 1. Effects of Scu and IL-1 $\beta$ on SW1353 cell viability. (A) Viability of SW1353 cells treated with increasing concentrations of Scu. (B) Scu attenuated

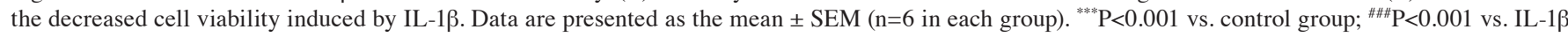
group. Scu, scutellarin; IL-1 $\beta$, interleukin $1 \beta$.
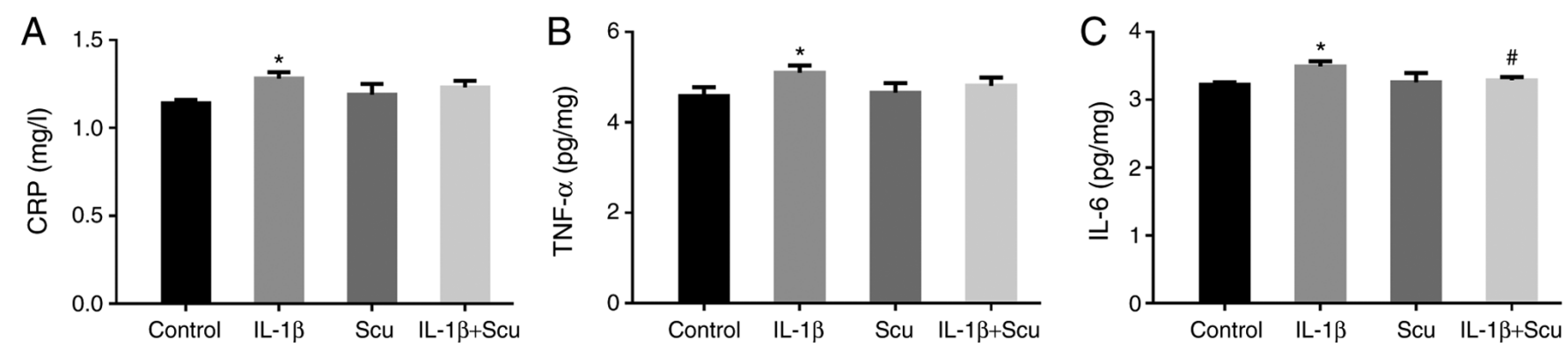

Figure 2. Effects of Scu on the expression of inflammatory cytokines. Changes in the expression of (A) CRP, (B) TNF- $\alpha$ and (C) IL-6 following treatment with Scu. Data are presented as the mean \pm SEM ( $n=3$ in each group). ${ }^{*} \mathrm{P}<0.05$ vs. control group; ${ }^{\#} \mathrm{P}<0.05$ vs. IL-1 $\beta$ group. Scu, scutellarin; CRP, C-reactive protein; TNF- $\alpha$, tumor necrosis factor- $\alpha$; IL- $1 \beta$, interleukin $1 \beta$.

Statistical analyses. GraphPad Prism v.6.0 (GraphPad Software, Inc.) was used to analyze the data generated in the charts in this experiment. All data are presented as the mean \pm SEM, and all experiments were performed three times. Differences between two groups were statistically analyzed using an unpaired, two-tailed Student's t-test. Differences among three groups were analyzed by one-way analysis of variance followed by Tukey's post hoc test. $\mathrm{P}<0.05$ was considered to indicate a statistically significant difference.

\section{Results}

Effects of scutellarin on SW1353 cell viability. To analyze the effects of scutellarin on human cartilage cells, the SW1353 cell line was used in the present study. A CCK-8 assay was used to measure the viability of SW1353 cells. As shown in Fig. 1A, no cell cytotoxicity was observed when the concentration of scutellarin was $<80 \mu \mathrm{mol} / \mathrm{l}$; however, cell viability was reduced when the concentration of scutellarin was $>80 \mu \mathrm{mol} / 1$. IL-1 $\beta$ was used to establish the model of OA using SW1353 cells; treatment of IL-1 $\beta$-induced SW1353 cells with scutellarin significantly increased cell viability (Fig. 1B).

Scutellarin affects the release of inflammatory cytokines, and the expression of collagen- and cholesterol-related proteins.
Western blot analysis was performed to investigate whether scutellarin inhibits the release of inflammatory cytokines, attenuates the degradation of collagen by regulating collagenrelated proteins, and reduces the level of cholesterol by regulating cholesterol-related proteins. In the present study (Fig. 2), the protein expression levels of CRP, TNF- $\alpha$ and IL-6 were measured. The results revealed that CRP, TNF- $\alpha$ and IL-6 expression was increased by IL-1 $\beta$; however, only the expression of IL-6 was significantly inhibited by scutellarin treatment (Fig. 2C).

The degradation of collagen is another feature of OA. As demonstrated in Fig. 3, the expression levels of $\mathrm{Col} 2$ and SOX 9 were significantly downregulated by IL- $1 \beta$, whereas MMP13 expression was upregulated. Scutellarin combined with IL-1 $\beta$ significantly increased the expression of $\mathrm{Col} 2$ and SOX9 (Fig. 3A-C), and significantly decreased the expression of MMP13 compared with the IL-1 $\beta$ group (Fig. 3D).

As aforementioned, high cholesterol level is another risk factor for OA. The present study measured the expression of $\mathrm{CH} 25 \mathrm{H}, \mathrm{CYP} 7 \mathrm{~B} 1, \mathrm{ABCA} 1$ and APOA-1 in SW1535 cells (Fig. 4). In the IL-1 $\beta$-induced group, the expression levels of $\mathrm{CH} 25 \mathrm{H}$ and CYP7B1 significantly increased, and those of ABCA1 and APOA-1 significantly decreased compared with the control group. Following treatment with scutellarin in the IL-1 1 -induced cell model, $\mathrm{CH} 25 \mathrm{H}$ and CYP7B1 expression levels were significantly downregulated (Fig. 4B and C). By 

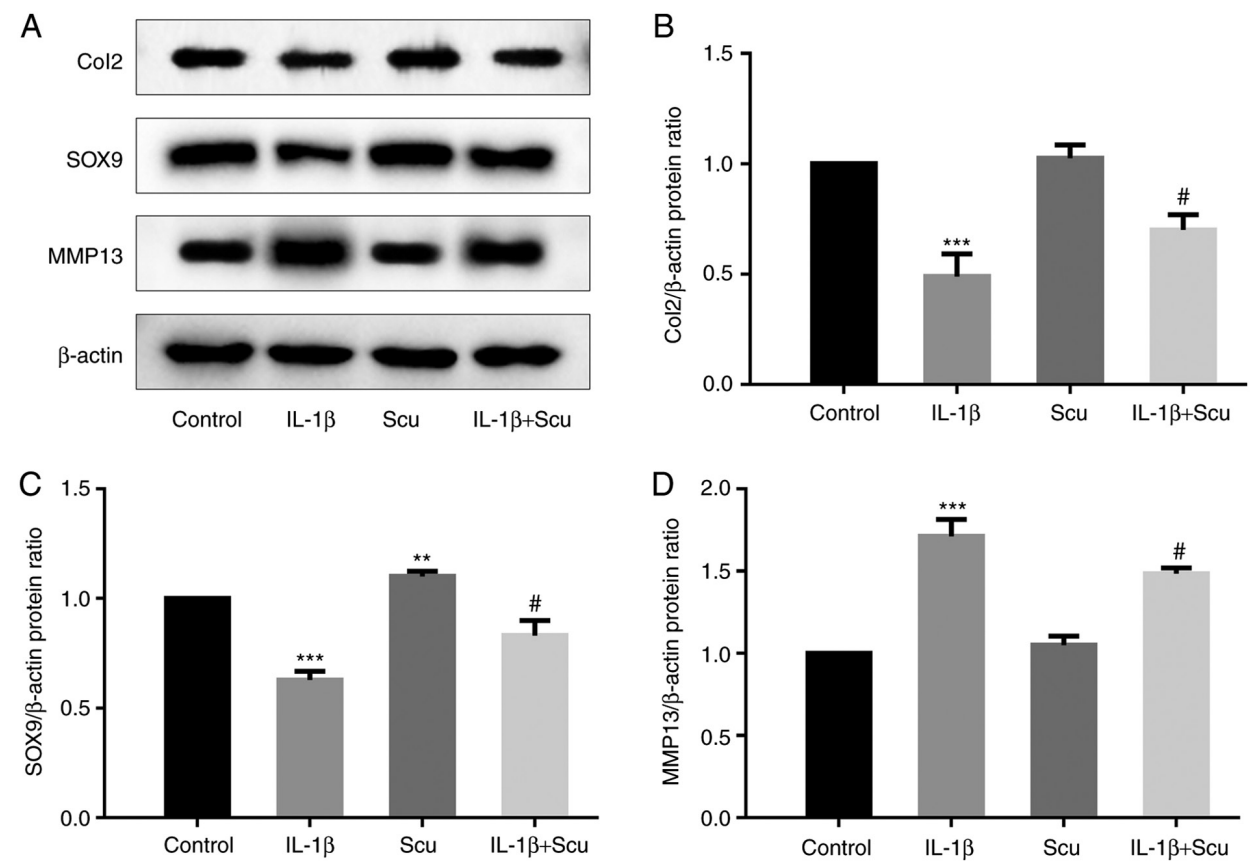

Figure 3. Scu regulates the expression of collagen-related proteins. (A) Collagen-related protein expression bands. Relative expression levels of (B) Col2, (C) SOX9 and (D) MMP13. Data are presented as the mean $\pm \mathrm{SEM}$ ( $\mathrm{n}=3$ in each group). ${ }^{* *} \mathrm{P}<0.01,{ }^{* * * *} \mathrm{P}<0.001$ vs. control group; ${ }^{*} \mathrm{P}<0.05$ vs. IL-1 $\beta$ group. Col2, collagen II; SOX9, SRY-box 9; MMP13, matrix metalloproteinase 13; Scu, scutellarin; IL-1 $\beta$, interleukin $1 \beta$.
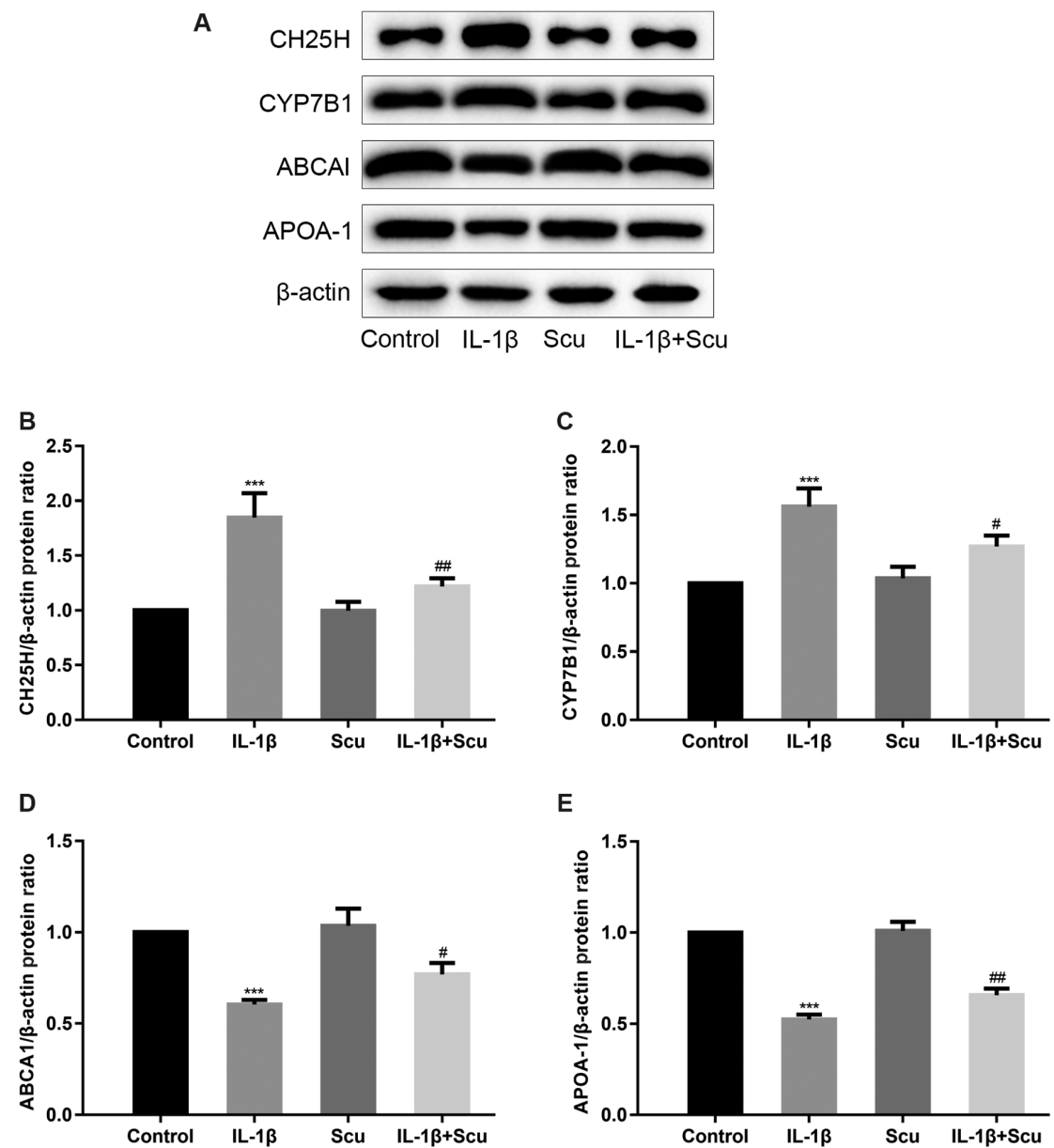

Figure 4. Scu regulates the expression of cholesterol-related proteins. (A) Cholesterol-related protein expression bands. Scu inhibited the IL-1 $\beta$-induced upregulation of (B) $\mathrm{CH} 25 \mathrm{H}$ and (C) CYP7B1 expression. The IL-1 1 -induced downregulation of (D) ABCA1 and (E) APOA-1 expression levels were elevated by Scu. Data are presented as the mean \pm SEM ( $n=3$ in each group). ${ }^{* * *} \mathrm{P}<0.001$ vs. control group; ${ }^{\#} \mathrm{P}<0.05,{ }^{\# \#} \mathrm{P}<0.05$ vs. IL-1 $\beta$ group. CH25H, cholesterol 25-hydroxylase; CYP7B1, cytochrome P450 family 7 subfamily B polypeptide 1; ABCA1, ATP-binding cassette transporter A1; APOA-1, apolipoprotein A-1; Scu, scutellarin; IL-1 $\beta$, interleukin $1 \beta$. 

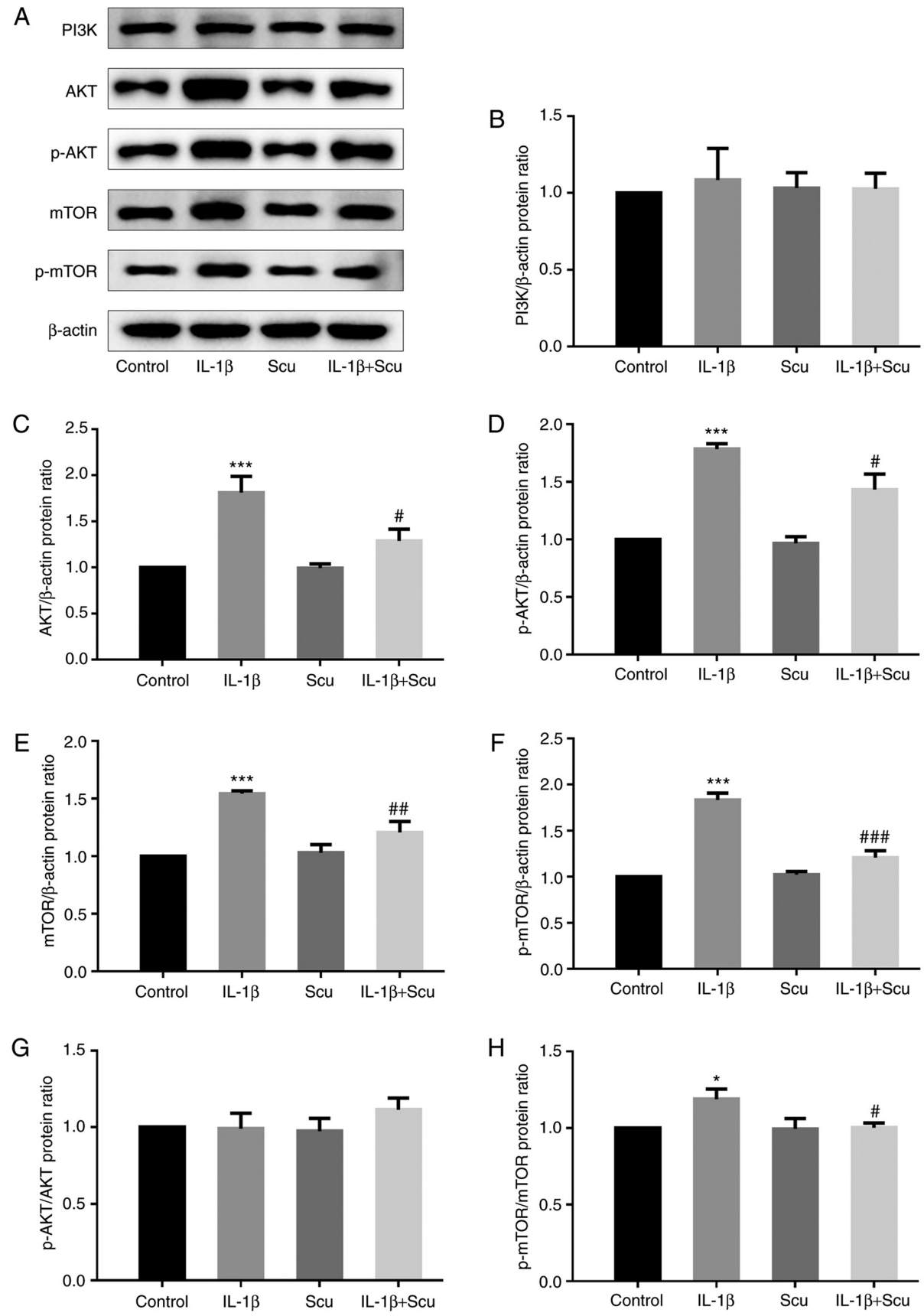

Figure 5. PI3K/AKT/mTOR signaling pathway in response to Scu treatment in IL-1ß-induced SW1535 cells. (A) Protein expression levels in the PI3K/AKT/mTOR signaling pathway were determined by western blotting. (B) The expression of PI3K exhibited no change following treatment with Scu and IL-1 $\beta$. Scu suppressed the IL-1 $\beta$-induced upregulation of (C) AKT, (D) p-AKT, (E) mTOR, (F) p-mTOR, (G) p-AKT/AKT and (H) p-mTOR/mTOR. Data are presented as the mean $\pm \operatorname{SEM}\left(\mathrm{n}=3\right.$ in each group). ${ }^{*} \mathrm{P}<0.05,{ }^{* * * *} \mathrm{P}<0.001$ vs. control group; ${ }^{*} \mathrm{P}<0.05,{ }^{\# \#} \mathrm{P}<0.01,{ }^{\# \# \#} \mathrm{P}<0.001$ vs. IL-1 $\beta$ group. $\mathrm{PI} 3 \mathrm{~K}$, phosphatidylinositol 3 kinase; p-, phosphorylated; mTOR, mammalian target of rapamycin; Scu, scutellarin; IL-1 $\beta$, interleukin $1 \beta$.

contrast, ABCA1 and APOA-1 expression levels significantly increased (Fig. 4D and E).

Scutellarin suppresses the PI3K/AKT/mTOR signaling pathway. In this experiment, the expression levels of AKT, $\mathrm{p}-\mathrm{AKT}, \mathrm{mTOR}$ and $\mathrm{p}-\mathrm{mTOR}$, and the ratio of $\mathrm{p}-\mathrm{mTOR} / \mathrm{mTOR}$ were increased by IL-1 $\beta$ (Fig. 5). Following treatment with scutellarin however, the levels of these proteins were significantly inhibited. Of note however, the expression of PI3K and the ratio of $\mathrm{p}-\mathrm{AKT} / \mathrm{AKT}$ remained unaltered among the groups (Fig. 5B and G). These results suggested that scutellarin inhibited the PI3K/AKT/mTOR signaling pathway.
Scutellarin and LY294002 treatment affect the release of inflammatory cytokines, and the expression of collagen- and cholesterol-related proteins. LY294002, as an inhibitor of $\mathrm{PI} 3 \mathrm{~K}$, was used to examine the effects of PI3K on the release of inflammatory cytokines, and the regulation of collagenand cholesterol-related proteins following treatment with scutellarin. Firstly, the expression levels of CRP, TNF- $\alpha$ and IL-6 were examined (Fig. 6), and only the level of IL-6 was significantly reduced by scutellarin and LY294002 (Fig. 6C). When compared with the IL- $1 \beta+$ scutellarin group, the addition of LY294002 significantly decreased the expression of IL-6. 

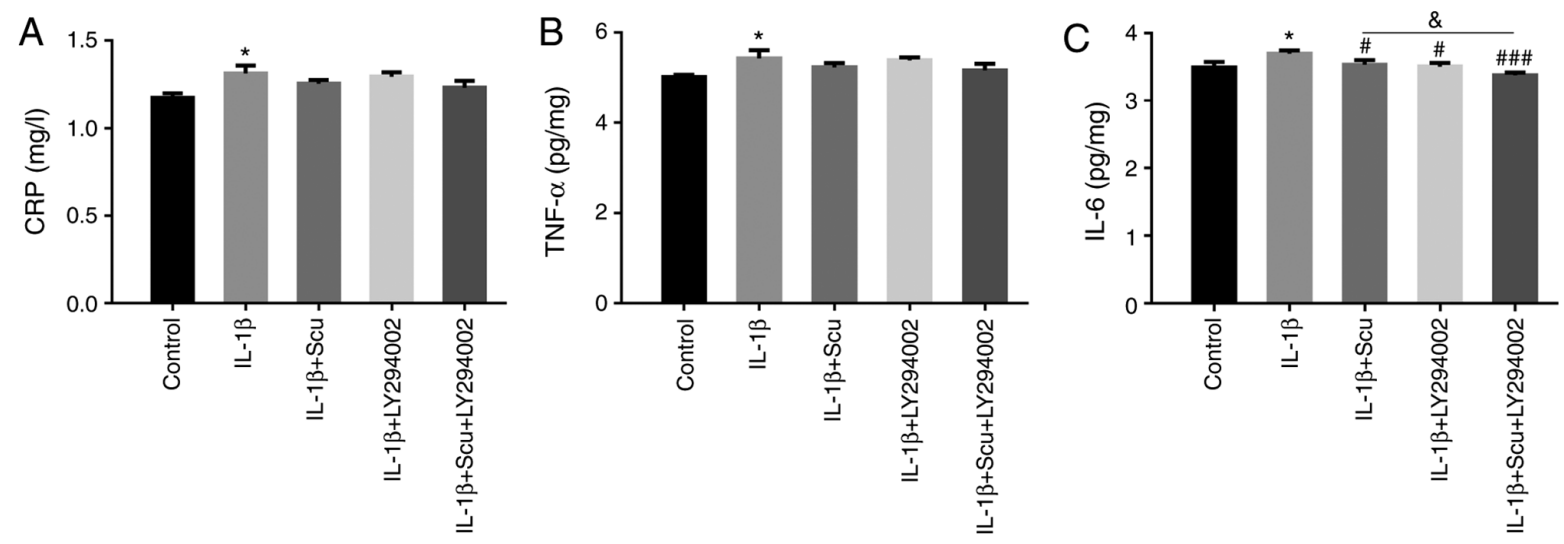

Figure 6. Effects of Scu and LY294002 on the release of inflammatory cytokines. Changes in the expression of (A) CRP, (B) TNF- $\alpha$ and (C) IL-6 following treatment with $\mathrm{Scu}$ and LY294002. Data are presented as the mean $\pm \mathrm{SEM}\left(\mathrm{n}=3\right.$ in each group). ${ }^{*} \mathrm{P}<0.05$ vs. control group; ${ }^{*} \mathrm{P}<0.05$, ${ }^{\# \# \#} \mathrm{P}<0.001$ vs. IL-1 $\beta$ group; ${ }^{\&} \mathrm{P}<0.05$ vs. the IL-1 $\beta+$ Scu + LY294002 group. CRP, C-reactive protein; TNF- $\alpha$, tumor necrosis factor- $\alpha$; IL-, interleukin; Scu, scutellarin.
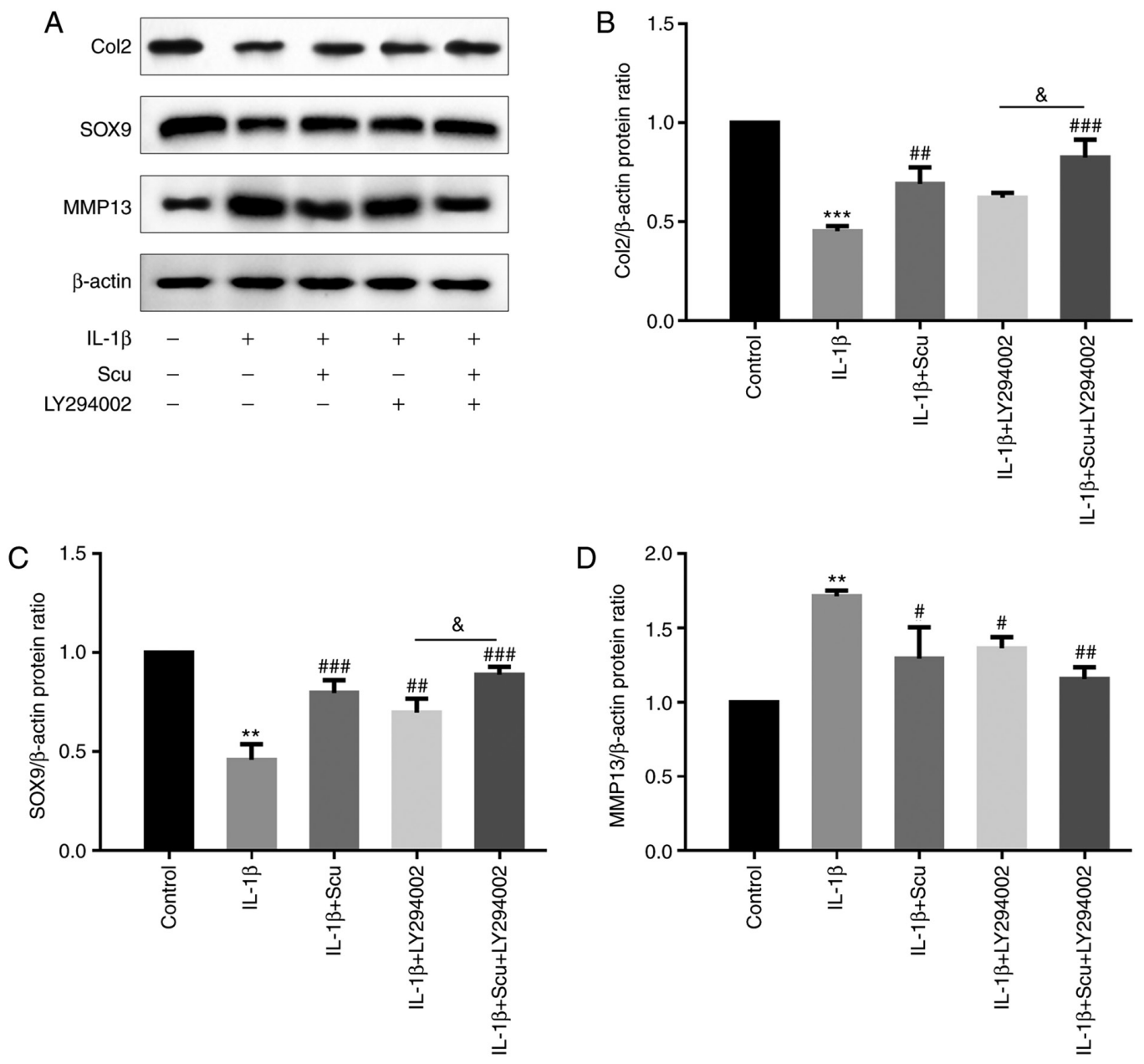

Figure 7. Scu regulates the expression of collagen-related proteins. (A) Collagen-related protein expression bands. Relative expression levels of (B) Col2, (C) SOX9 and (D) MMP13. Data are presented as the mean \pm SEM ( $\mathrm{n}=3$ in each group). ${ }^{* *} \mathrm{P}<0.01,{ }^{* * *} \mathrm{P}<0.001$ vs. control group; ${ }^{*} \mathrm{P}<0.05$, ${ }^{\# \#} \mathrm{P}<0.01,{ }^{\# \# "} \mathrm{P}<0.001$ vs. IL-1 $\beta$ group; ${ }^{\circledR} \mathrm{P}<0.05$ vs. IL-1 + Scu + LY294002 group. Co12, collagen II; SOX9, SRY-box 9; MMP13, matrix metalloproteinase 13; Scu, scutellarin; IL-1 $\beta$, interleukin $1 \beta$.

In regard to the collagen-related proteins (Fig. 7), the IL-1 $\beta$-induced increase in MMP13 expression was decreased by treatment with scutellarin and LY294002, and the greatest decrease was observed in the group treated with scutellarin and LY294002 in combination (Fig. 7D). By contrast, compared with the IL-1 $\beta$-induced model group, the expression of Col2 (Fig. 7B) and SOX9 (Fig. 7C) increased following treatment with scutellarin and LY294002. When compared with the IL-1 $\beta+$ LY294002 group, the addition of scutellarin significantly increased the expression of $\mathrm{Col} 2$ and SOX9. 

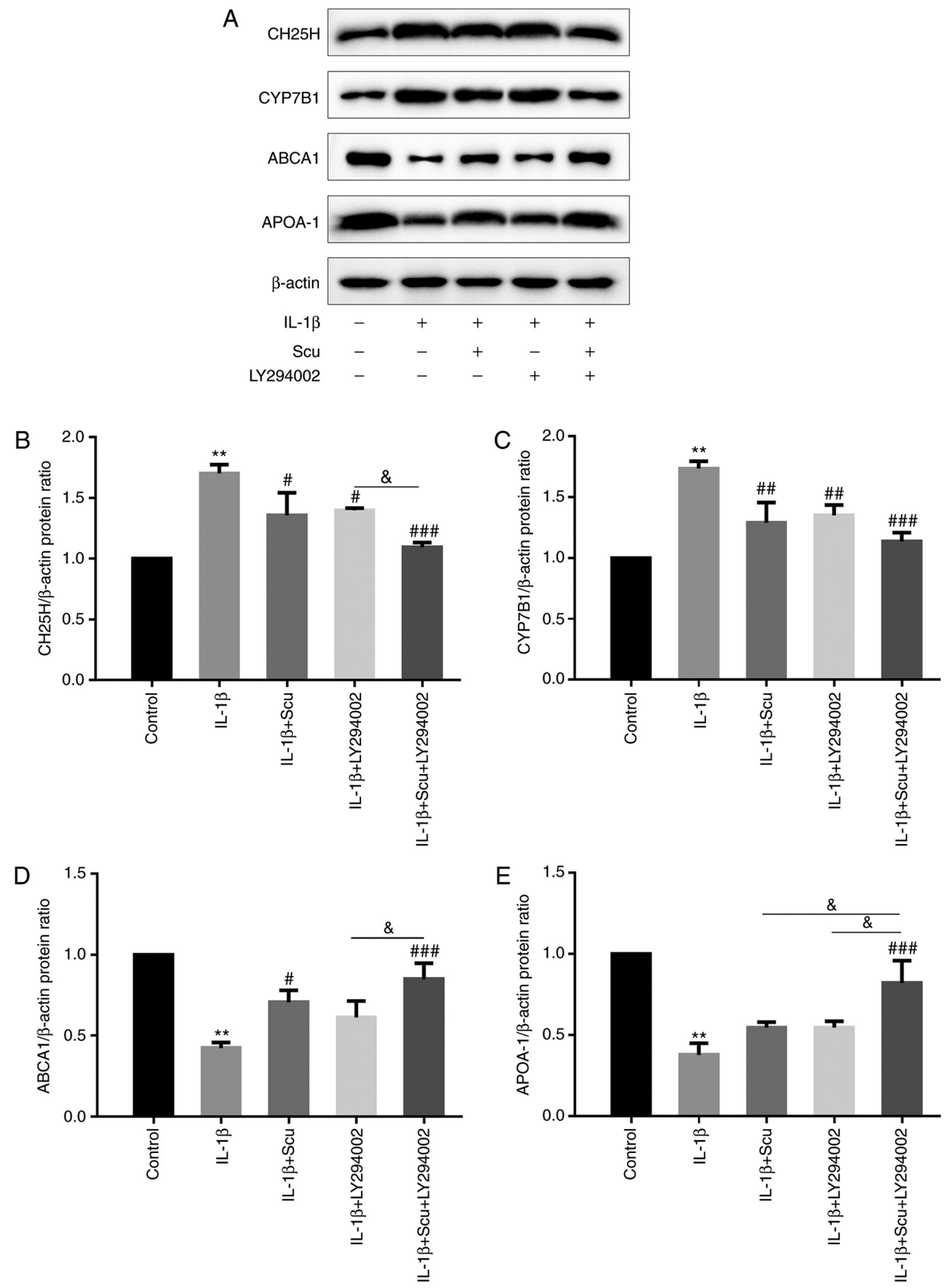

Figure 8. Scu regulates the expression of cholesterol-related proteins. (A) Cholesterol-related protein expression bands. Scu and LY294002 inhibited the IL-1ßinduced upregulation of (B) $\mathrm{CH} 25 \mathrm{H}$ and (C) CYP7B1 expression. The IL-1 $\beta$-induced downregulation of (D) ABCA1 and (E) APOA-1 expression was elevated

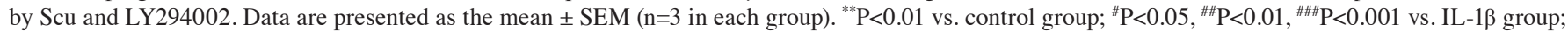
${ }^{\&} \mathrm{P}<0.05$ vs. IL-1 $\beta+$ Scu + LY294002 group. CH25H, cholesterol 25-hydroxylase; CYP7B1, cytochrome P450 family 7 subfamily B polypeptide 1; ABCA1, ATP-binding cassette transporter A1; APOA-1, apolipoprotein A-1; Scu, scutellarin; IL-1 $\beta$, interleukin $1 \beta$.

Thus, scutellarin and LY294002 can partially restore the loss of Col2 and SOX9 caused by IL-1 $\beta$.

The expression levels of cholesterol-related proteins were also regulated by scutellarin and LY294002 (Fig. 8). The IL-1 $\beta$ induced upregulation of $\mathrm{CH} 25 \mathrm{H}$ and $\mathrm{CYP} 7 \mathrm{~B} 1$ expression was reversed by scutellarin and LY294002 (Fig. 8B and C), whereas the IL-1 $\beta$-induced downregulation of ABCA1 and APOA-1 expression was reversed by a combination of scutellarin and LY294002 (Fig. 8D and E).
Inhibition of the PI3K/AKT/mTOR signaling pathway by scutellarin and LY294002. To directly examine the effect of scutellarin and LY294002 on the PI3K/AKT/mTOR signaling pathway, the expression levels of PI3K, AKT, p-AKT, mTOR and p-mTOR were determined (Fig. 9). The results of the western blot analysis revealed that scutellarin and LY294002 affected the expression of AKT, mTOR, and the ratio of $\mathrm{p}-\mathrm{mTOR} / \mathrm{mTOR}$, but not that of $\mathrm{PI} 3 \mathrm{~K}$ and the ratio of p-AKT/AKT. In combination, LY294002 and scutellarin 
A
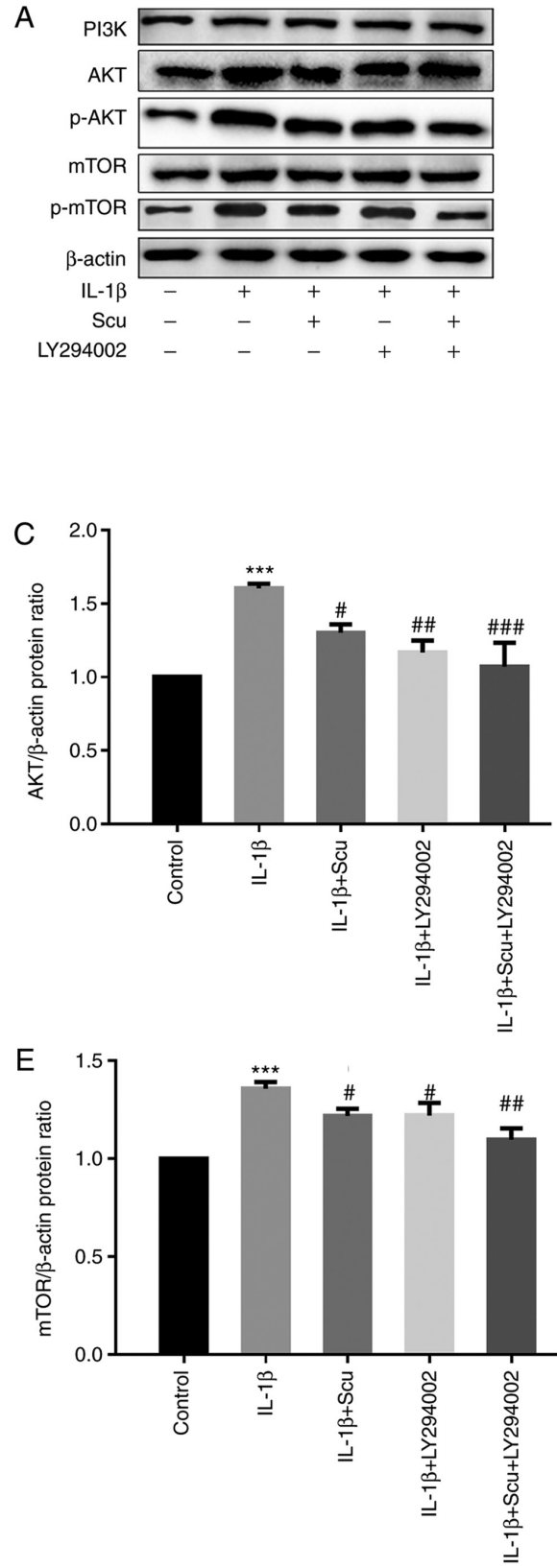
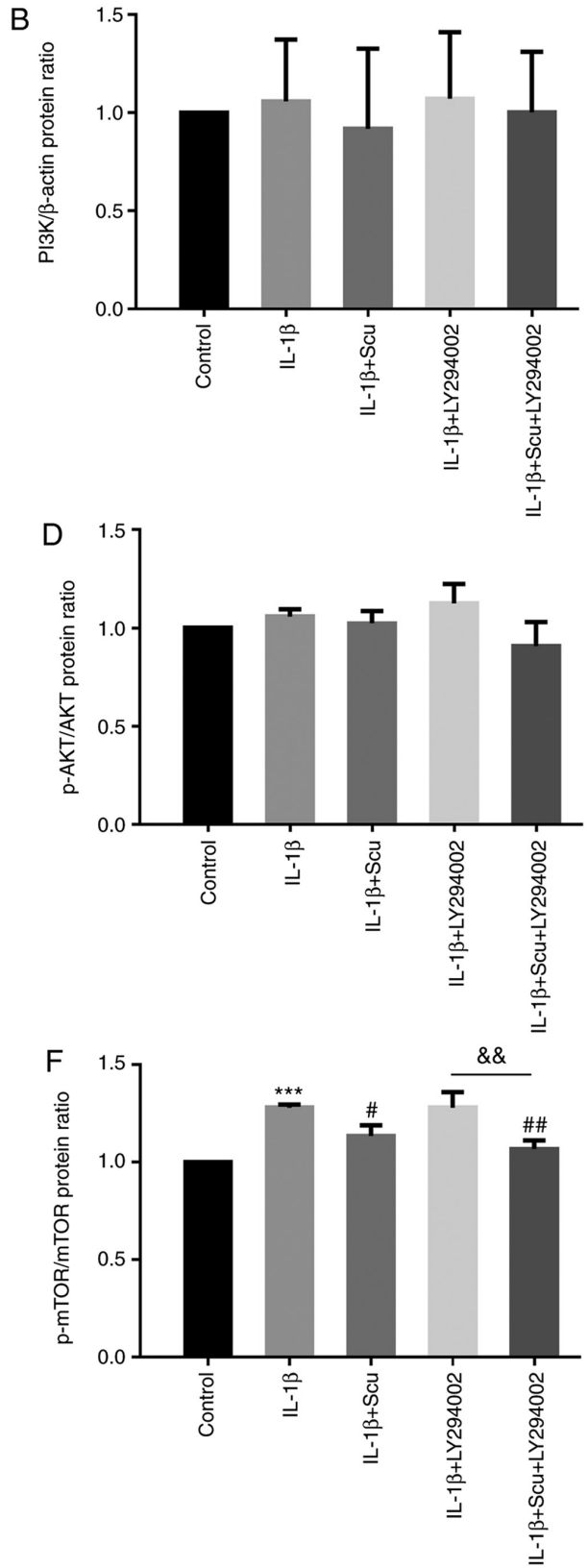

Figure 9. PI3K/AKT/mTOR signaling pathway in response to Scu and LY294002 in IL-1ß-induced SW1535 cells. (A) Protein expression bands of PI3K, AKT, p-AKT, mTOR and p-mTOR. (B) The protein expression of PI3K was not significantly different among the groups. (C) Scu and LY294002 treatment inhibited the expression of AKT. (D) The ratio of p-AKT/AKT was not significantly different among the groups. (E) Scu and LY294002 treatment inhibited the expression of mTOR. (F) Scu and LY294002 treatment inhibited the ratio of $\mathrm{p}-\mathrm{mTOR} / \mathrm{mTOR}$. ${ }^{* * *} \mathrm{P}<0.001$ vs. control group; ${ }^{\#} \mathrm{P}<0.05,{ }^{\# \#} \mathrm{P}<0.01,{ }^{\# \# \#} \mathrm{P}<0.001$ vs. IL-1 group; \&\&P<0.01. PI3K, phosphatidylinositol 3 kinase; p-, phosphorylated; mTOR, mammalian target of rapamycin; Scu, scutellarin; IL-1 $\beta$, interleukin $1 \beta$.

decreased the IL-1 $\beta$-induced upregulation of AKT, mTOR and the ratio of p-mTOR/mTOR (Fig. 9C, E and F). Thus, this indicated that scutellarin and LY294002 are able to inactivate the PI3K/AKT/mTOR signaling pathway.

\section{Discussion}

$\mathrm{OA}$, as a chronic disease that results in disability and a reduced quality of life, results from several different etiologies (25). A number of studies have been performed to elucidate the mechanisms responsible and to identify novel treatment strategies for OA (2). Acetaminophen, a non-steroidal anti-inflammatory drug, and corticosteroids as oral drugs, are common pharmacological therapies for the management of OA. However, they may cause toxicity when at high doses and are associated with adverse reactions, such as gastric ulceration, kidney dysfunction and increased bleeding under particular conditions $(3,26)$. Thus, the development of effective drugs with limited side effects is of utmost importance. Recently, Chinese Traditional Medicine has gained increasing attention. Effective components of Erigeron breviscapus, including scutellarin, have been reported to possess anti-inflammatory properties (27) and to restrain the development of tumors (22). In the present study, the effects of scutellarin on the PI3K/AKT/mTOR signaling pathway were examined, and it was determined whether it can inhibit the inflammatory response, and the expression of collagen- and cholesterol-related proteins in the SW1353 cell model of OA. 
$\mathrm{OA}$ is regarded as an inflammatory disease (6). Several inflammatory cytokines are secreted during OA, and biomarkers of inflammation, including CRP and TNF- $\alpha$ are elevated. IL-6 is an inflammatory cytokine that is associated with systemic low-grade inflammation, and its expression is also altered in OA (28). Scutellarin has been reported to inhibit the expression of inflammatory factors, such as IL-1 $\beta$, IL-6 and TNF- $\alpha$ in collagen-induced arthritis (29). Another study reported that treatment with scutellarin downregulated the expression levels of IL-1 $\beta$, TNF- $\alpha$ and IL- 6 in the serum of osteoarthritis mice (30). In the present study, it was found that only the expression of IL- 6 was influenced by scutellarin. The levels of CRP and TNF- $\alpha$ were not altered. Naderi et al (31) measured the effects of Zingiber officinale on patients with knee osteoarthritis, and no changes in the levels of CRP were found within 3 months. Another study demonstrated that the expression of CRP had no association with the incidence or progression of OA (32). Besides, in the present study, scutellarin failed to inhibit the IL- $1 \beta$-induced increase in TNF- $\alpha$ expression. In fact, unlike the chondroid cells isolated from patients and in vivo animal models, the expression levels of CRP, TNF- $\alpha$ and IL- 6 were all only slightly increased by IL-1 $\beta$ in SW1353 cells. This was a limitation of the present research. Although the SW1353 cell line used to establish the cell model of OA has been reported on by some previous research (33-35) because the cell lines could provide enough and steady cells for the research, the IL-1 $\beta$-induced OA cell model used in the present study cannot completely mimic the in vivo environment. This may partly explain why there were no changes in the expression levels of CRP and TNF- $\alpha$ in this study.

In OA, the expression of $\mathrm{Col} 2$ and $\mathrm{SOX} 9$ are influenced by MMP13. As reported by Otero et al (36), the expression of ETS-related transcription factor Elf-3 protein driven by MMP13 was increased in OA-affected cartilage, and it inhibited SOX9-driven COL2A1 promoter activity. Ouyang et al (37) also found decreased expression of $\mathrm{Col} 2$ and SOX9, but increased expression of MMP13 in OA. Thus, indicating that the expression of MMP13 is enhanced, whereas that of Col2 and SOX9 is inhibited in OA. Fisetin is a polyphenol extracted from fruit and vegetables, and it can inhibit the IL-1 $\beta$-induced upregulation of MMP13 expression and decrease the degradation of Col2 and SOX9 in OA (38). Scutellarin has been demonstrated to have similar functions; the mRNA expression of MMP13 was found to be decreased, whereas Col2 expression was increased by treatment with scutellarin in OA mouse models (30). These findings were supported by those of another study in human primary chondrocytes (24). In the present study, similar results were obtained. The expression of $\mathrm{Col} 2$ and SOX9 increased and the expression of MMP13 decreased by treatment with scutellarin. These results suggested that scutellarin can attenuate the degradation of cartilage in OA.

The function of scutellarin in regulating cholesterol-related protein expression in the OA cell model was determined in the present study. The results revealed that scutellarin downregulated the expression of $\mathrm{CH} 25 \mathrm{H}$ and $\mathrm{CYP} 7 \mathrm{~B} 1$ and upregulated the expression of ABCA1 and APOA-1. As a metabolic disorder disease (39), it has been demonstrated that cholesterol metabolism is abnormal in OA with increased levels of LDL (40). Tsezou et al (41) reported that APOA-1 and
ABCA1 expression decreased in OA-affected cartilage. On the other hand, the expression levels of $\mathrm{CH} 25 \mathrm{H}$ and $\mathrm{CYP} 7 \mathrm{~B} 1$ have been found to be increased, leading to high levels of cholesterol (42). The findings of the present study were consistent with these previous findings, therefore demonstrating that scutellarin can decrease total cholesterol levels and improve lipid metabolism $(43,44)$.

The PI3K/AKT/mTOR signaling pathway has been found to be associated with OA $(22,45)$, and the inhibition of this pathway can attenuate inflammation and articular cartilage degeneration in OA (8-10). In the present study, no significant changes were identified in the protein expression of PI3K between the groups, which indicated that IL-1 $\beta$, scutellarin and LY294002 may not affect the expression of PI3K. The present study also found that scutellarin inhibited the expression of AKT, mTOR and p-mTOR, and the ratio of p-mTOR/ mTOR in the IL-1 $\beta$-induced cells. The ratio of p-AKT /AKT may not have been affected because the decrease in p-AKT expression may be caused by the decrease in AKT expression and the decrease in AKT expression essentially leads to the decrease in mTOR activity. It has been revealed that the activation of PI3K/AKT signaling can degrade chondrocytes and accelerate the progression of OA (8-10). Thus, these findings suggested that scutellarin can attenuate OA by regulating the $\mathrm{PI} 3 \mathrm{~K} / \mathrm{AKT} / \mathrm{mTOR}$ signaling pathway.

In conclusion, the primary finding of the present study was that scutellarin inhibited the activation of the PI3K/AKT/mTOR signaling pathway, which alleviated cartilage degradation by regulating relevant protein levels, and decreased cholesterol levels by regulating cholesterol-related protein expression in the OA cell model; however, it only affected the release of the inflammatory cytokine, IL-6. These findings are important for future studies investigating the function of scutellarin in OA and they provide evidence that scutellarin could be used as a latent medicine for OA. However, further studies are required to confirm the pharmacological functions of scutellarin in vivo and to explore the precise underlying mechanisms.

\section{Acknowledgements}

Not applicable.

\section{Funding}

The present study was financially supported by the Application Foundation Fund of Science and Technology Department of Sichuan Province (grant no. 2018JY0264), Innovative topics of Chengdu Sport University (grant no. CX19C01) and Innovation team of Chengdu Sport University (grant no. CXTD201805).

\section{Availability of data and materials}

The datasets used and/or analyzed during the current study are available from the corresponding author upon reasonable request.

\section{Authors' contributions}

SHJ, BXH and LRT designed the study. SHJ, PWL and YLT performed the experiments. YTZ, XHL and MJW analyzed 
the data. SHJ wrote the manuscript. All authors critically reviewed the manuscript, and read and approved the final manuscript.

\section{Ethics approval and consent to participate}

Not applicable.

\section{Patient consent for publication}

Not applicable.

\section{Competing interests}

The authors declare that they have no competing interests.

\section{References}

1. Pereira D, Ramos E and Branco J: Osteoarthritis. Acta Med Port 28: 99-106, 2015.

2. Nelson AE: Osteoarthritis year in review 2017: Clinical Osteoarthritis Cartilage 26: 319-325, 2018.

3. Saccomano SJ: Osteoarthritis treatment: Decreasing pain, improving mobility. Nurse Pract 43: 49-55, 2018.

4. Palazzo C, Nguyen C, Lefevre-Colau MM, Rannou F and Poiraudeau S: Risk factors and burden of osteoarthritis. Ann Phys Rehabil Med 59: 134-138, 2016.

5. Vina ER and Kwoh CK: Epidemiology of osteoarthritis: Literature update. Curr Opin Rheumatol 30: 160-167, 2018.

6. Berenbaum F: Osteoarthritis as an inflammatory disease (osteoarthritis is not osteoarthrosis!). Osteoarthritis Cartilage 21: 16-21, 2013.

7. Farnaghi S, Crawford R, Xiao Y and Prasadam I: Cholesterol metabolism in pathogenesis of osteoarthritis disease. Int J Rheum Dis 20: 131-140, 2017.

8. Xue JF, Shi ZM, Zou J and Li XL: Inhibition of PI3K/AKT/mTOR signaling pathway promotes autophagy of articular chondrocytes and attenuates inflammatory response in rats with osteoarthritis Biomed Pharmacother 89: 1252-1261, 2017.

9. Lin C, Shao Y, Zeng C, Zhao C, Fang H, Wang L, Pan J, Liu L, Qi W, Feng X, et al: Blocking PI3K/AKT signaling inhibits bone sclerosis in subchondral bone and attenuates post-traumatic osteoarthritis. J Cell Physiol 233: 6135-6147, 2018.

10. Chen J, Crawford R and Xiao Y: Vertical inhibition of the $\mathrm{PI} 3 \mathrm{~K} / \mathrm{Akt} / \mathrm{mTOR}$ pathway for the treatment of osteoarthritis. J Cell Biochem 114: 245-249, 2013.

11. Farnaghi S, Crawford R, Xiao Y and Prasadam I: Cholestero metabolism in pathogenesis of osteoarthritis disease. Int J Rheum 20: 131-140, 2017.

12. de Munter W, van der Kraan PM, van den Berg WB and van Lent PL: High systemic levels of low-density lipoprotein cholesterol: Fuel to the flames in inflammatory osteoarthritis? Rheumatology 55: 16-24, 2016.

13. Beier F: Cholesterol and cartilage do not mix well. Nat Rev Rheumatol 15: 253-254, 2019.

14. Varshney P and Saini N: PI3K/AKT/mTOR activation and autophagy inhibition plays a key role in increased cholesterol during IL-17A mediated inflammatory response in psoriasis. Biochim Biophys Acta Mol Basis Dis 1864 (5 Pt A): 1795-1803, 2018.

15. Fuentes M, Santander N and Cortés V: Insulin increases cholesterol uptake, lipid droplet content, and apolipoprotein B secretion in $\mathrm{CaCo}-2$ cells by upregulating SR-BI via a PI3K, AKT, and mTOR-dependent pathway. J Cell Biochem: October 2, 2018 (Online ahead of print). doi: 10.1002/jcb.27410.

16. Yang $\mathrm{Y}, \mathrm{Gu} \mathrm{Y}$, Zhao $\mathrm{H}$ and Zhang S: Loganin attenuates osteoarthritis in rats by inhibiting IL-1 $\beta$-induced catabolism and apoptosis in chondrocytes via regulation of phosphatidylinositol 3-lkinases (PI3K)/Akt. Med Sci Monit 25: 4159-4168, 2019.

17. Li X, Wu D, Hu Z, Xuan J, Ding X, Zheng G, Feng Z, Ni W and Wu A: The protective effect of ligustilide in osteoarthritis: An in vitro and in vivo study. Cell Physiol Biochem 48: 2583-2595, 2018.
18. Pan T, Cheng TF, Jia YR, Li P and Li F: Anti-rheumatoid arthritis effects of traditional Chinese herb couple in adjuvant-induced arthritis in rats. J Ethnopharmacol 205: 1-7, 2017.

19. Salimzadeh A, Ghourchian A, Choopani R, Hajimehdipoor H, Kamalinejad M and Abolhasani M: Effect of an orally formulated processed black cumin, from Iranian traditional medicine pharmacopoeia, in relieving symptoms of knee osteoarthritis: A prospective, randomized, double-blind and placebo-controlled clinical trial. Int J Rheum Dis 20: 691-701, 2017.

20. Xu X, Lv H, Li X, Su H, Zhang X and Yang J: Danshen attenuates osteoarthritis-related cartilage degeneration through inhibition of NF-kappaB signaling pathway in vivo and in vitro. Bio Cell Biol 95: 644-651, 2017.

21. Wang L and Ma Q: Clinical benefits and pharmacology of scutellarin: A comprehensive review. Pharmacol Ther 190: 105-127, 2018.

22. Li CY, Wang Q, Wang X, Li G, Shen S and Wei X: Scutellarin inhibits the invasive potential of malignant melanoma cells through the suppression epithelial-mesenchymal transition and angiogenesis via the PI3K/Akt/mTOR signaling pathway. Eur J Pharmacol 858: 172463, 2019.

23. Lv WL, Liu Q, An JH and Song XY: Scutellarin inhibits hypoxiainduced epithelial-mesenchymal transition in bladder cancer cells. J Cell Physiol 234: 23169-23175, 2019.

24. Liu F, Li L, Lu W, Ding Z, Huang W, Li YT, Cheng C, Shan WS $\mathrm{Xu} \mathrm{J}, \mathrm{He} \mathrm{W}$, et al: Scutellarin ameliorates cartilage degeneration in osteoarthritis by inhibiting the Wnt/ $\beta$-catenin and MAPK signaling pathways. Int Immunopharmacol 78: 105954, 2020

25. Lane NE, Shidara K and Wise BL: Osteoarthritis year in review 2016: Clinical. Osteoarthritis Cartilage 25: 209-215, 2017.

26. Zhang W, Ouyang H, Dass CR and Xu J: Current research on pharmacologic and regenerative therapies for osteoarthritis. Bone Res 4: 15040, 2016

27. Luo P, Zhang Z, Yi T, Zhang H, Liu X and Mo Z: Antiinflammatory activity of the extracts and fractions from Erigeron multiradiatus through bioassay-guided procedures. J Ethnopharmacol 119: 232-237, 2008.

28. Watt FE: Osteoarthritis biomarkers: Year in review. Osteoarthritis Cartilage 26: 312-318, 2018

29. Zhang L, Sun S, Li W, Zhang W, Wang X and Yang SY: Effect of Scutellarin inhibits collagen-induced arthritis through TLR4/NF- $\mathrm{BB}$-mediated inflammation. Mol Med Rep 16: 5555-5560, 2017.

30. Wang W, Li J, Li F, Peng J, Xu M, Shangguan Y, Li Y, Zhao Y, Qiu C, Qu R, et al: Scutellarin suppresses cartilage destruction in osteoarthritis mouse model by inhibiting the $\mathrm{NF}-\kappa \mathrm{B}$ and PI3K/AKT signaling pathways. Int Immunopharmacol 77: 105928, 2019

31. Naderi Z, Mozaffari-Khosravi H, Dehghan A, Nadjarzadeh A and Huseini HF: Effect of ginger powder supplementation on nitric oxide and $\mathrm{C}$-reactive protein in elderly knee osteoarthritis patients: A 12-week double-blind randomized placebo-controlled clinical trial. J Tradit Complement Med 6: 199-203, 2015.

32. Kerkhof HJ, Bierma-Zeinstra SM, Castano-Betancourt MC, de Maat MP, Hofman A, Pols HA, Rivadeneira F, Witteman JC, Uitterlinden AG and van Meurs JB: Serum C reactive protein levels and genetic variation in the CRP gene are not associated with the prevalence, incidence or progression of osteoarthritis independent of body mass index. Ann Rheum Dis 69: 1976-1982, 2010.

33. Xu X, Liu X, Yang Y, He J, Gu H, Jiang M, Huang Y, Liu X and Liu L: Resveratrol inhibits the development of obesity-related osteoarthritis via the TLR4 and PI3K/Akt signaling pathways. Connect Tissue Res 60: 571-582, 2019.

34. Gao F and Zhang S: Salicin inhibits AGE-induced degradation of type II collagen and aggrecan in human SW1353 chondrocytes: Therapeutic potential in osteoarthritis. Artif Cells Nanomed Biotechnol 47: 1043-1049, 2019.

35. Hong GU, Lee JY, Kang H, Kim TY, Park JY, Hong EY, Shin YH, Jung SH, Chang HB, Kim YH, et al: Inhibition of osteoarthritiseelated molecules by isomucronulatol $7-O-\beta$-d-glucoside and Ecliptasaponin A in IL-1 $\beta$-stimulated chondrosarcoma cell model. Molecules 23: 2807, 2018. doi: 10.3390/molecules23112807.

36. Otero M, Peng H, Hachem KE, Culley KL, Wondimu EB, Quinn J, Asahara $\mathrm{H}$, Tsuchimochi $\mathrm{K}$, Hashimoto $\mathrm{K}$ and Goldring MB: ELF3 modulates type II collagen gene (COL2A1) transcription in chondrocytes by inhibiting SOX9-CBP/p300-driven histone acetyltransferase activity. Connect Tissue Res 58: 15-26, 2017.

37. Ouyang Y, Wang W, Tu B, Zhu Y, Fan C and Li Y: Overexpression of SOX9 alleviates the progression of human osteoarthritis in vitro and in vivo. Drug Des Devel Ther 13: 2833-2842, 2019. 
38. Zheng W, Feng Z, You S, Zhang H, Tao Z, Wang Q, Chen H and $\mathrm{Wu}$ Y: Fisetin inhibits IL-1 $\beta$-induced inflammatory response in human osteoarthritis chondrocytes through activating SIRT1 and attenuates the progression of osteoarthritis in mice. Int Immunopharmacol 45: 135-147, 2017.

39. Mobasheri A, Rayman MP, Gualillo O, Sellam J, van der Kraan P and Fearon U: The role of metabolism in the pathogenesis of osteoarthritis. Nat Rev Rheumatol 13: 302-311, 2017.

40. de Munter W, van den Bosch MH, Slöetjes AW, Croce KJ, Vogl T, Roth J, Koenders MI, van de Loo FA, van den Berg WB, van der Kraan PM, et al: High LDL levels lead to increased synovial inflammation and accelerated ectopic bone formation during experimental osteoarthritis. Osteoarthritis Cartilage 24: 844-855, 2016.

41. Tsezou A, Iliopoulos D, Malizos KN and Simopoulou T: Impaired expression of genes regulating cholesterol efflux in human osteoarthritic chondrocytes. J Orthop Res 28: 1033-1039, 2010.
42. Choi WS, Lee G, Song WH, Koh JT, Yang J, Kwak JS, Kim HE, Kim SK, Son YO, Nam H, et al: The CH25H-CYP7B1-RORa axis of cholesterol metabolism regulates osteoarthritis. Nature 566: 254-258, 2019.

43. Li Q, Wu JH, Guo DJ, Cheng HL, Chen SL and Chan SW: Suppression of diet-induced hypercholesterolemia by scutellarin in rats. Planta Med 75: 1203-1208, 2009.

44. Fan H, Ma X, Lin P, Kang Q, Zhao Z, Wang L, Sun D, Cheng J and $\mathrm{Li} \mathrm{Y}$ : Scutellarin prevents nonalcoholic fatty liver disease (NAFLD) and hyperlipidemia via PI3K/AKT-dependent activation of nuclear factor (erythroid-derived 2)-like 2 (Nrf2) in rats. Med Sci Monit 23: 5599-5612, 2017.

45. Tang SL, Gao YL and Hu WZ: Scutellarin inhibits the metastasis and cisplatin resistance in glioma cells. OncoTargets Ther 12: 587-598, 2019. 